\title{
What is Business Process Management: A Two Stage Literature Review of an Emerging Field
}

\author{
Charles Møller' ${ }^{1}$ Carsten J. Maack ${ }^{2}$ and Rune D. Tan ${ }^{3}$ \\ 'Aalborg University, DK-9220 Aalborg charles@production.aau.dk \\ ${ }^{2}$ Aarhus School of Business, DK-8210 Aarhus V carsten@ma2ck.dk \\ ${ }^{3}$ Vestas Wind Systems A/S, DK-8900 Randers rdtan@vestas.com
}

\begin{abstract}
Business Process Management (BPM) is an emerging new field in business. However there is no academically agreed upon conceptual framework. The aim of this paper is to establish a conceptual framework grounded in the recent literature. The purpose of this work is to ensure a better foundation for future research and to discussion of the implications of BPM on Enterprise Information Systems (EIS). The starting point of this study is a focused literature review of the BPM concept. This literature review leads to the formulation of a conceptual framework for BPM which is evaluated using a quantitative lexical analysis of a broader literature sample. Finally the implication of the BPM on EIS is discussed and potential future research opportunities are outlined.
\end{abstract}

Keywords: Business process management (BPM), Work flow management, Business transformation

\section{INTRODUCTION}

Business Process Management (BPM) is a new concept that is gaining an increased recognition in the management literature $[1,2]$. Recently we have seen that traditional computer science research in workflow management is extending its perspective towards BPM [3]. However there is no academically agreed upon conceptual framework for BPM, and the concept of BPM is often used for commercial purposes, which makes it hard to grasp the fundamental idea of BPM [4]. Analyst Terry Schurter defines (BPM) as a natural and holistic management approach to operating business that produces a highly efficient, agile, innovative, and adaptive organization that far exceeds that achievable through traditional management approaches [5]. This definition makes no explicit reference to IT, but rather agility is pivotal. Agility is vital to contemporary business and the role of IT in shaping agility is obvious [6]. Consequently a common fframework across business, technology and Information Systems (IS) is needed.

BPM is often proclaimed as a new management principle that gives companies the competitive advantages that is needed to support more agile and flexible business processes. Enterprise Information Systems (EIS), on the other hand, are described as tightly coupled system because they are built upon specific reference data models and process models, but still they are claimed inadequate to support agile business

Please use the following format when citing this chapter:

Møller, C., Maack, C. J., Tan, R. D., 2007, in IFIP International Federation for Information Processing, Volume 254, Research and Practical Issues of Enterprise Information Systems II Volume 1, eds. I. Xu. Tjoa A.. Chaudhry S. (Boston: Springer), pp. 19-31. 
processes. However, contemporary EIS is rapidly evolving towards BPM oriented principles [7]. The lack of agility and flexibility is thus the Achilles heel of many EIS. BPM appears to be the solution to this problem, but what is BPM really about and what are the implications to EIS? Therefore the aim of the paper is to present a comprehensive study and common framework for the concept of BPM in an EIS perspective. The purpose of this work is to ensure a better foundation for future research and discussion of the implications of BPM on EIS. The starting point of this study is a focused literature review of the BPM concept. The research methodology is discussed in the next section. This literature review presented in the next chapter leads to the formulation of a conceptual framework for BPM which is evaluated using a quantitative lexical analysis of a broader literature sample. Finally the implication of the BPM on EIS is discussed and potential future research opportunities are outlined.

\subsection{Methodology}

The analysis of BPM and the preparation of this paper is done on basis of the tree books: [1], [3] and central chapters of [2]. These books have contributed to the understanding of the conceptual terms in the BPM literature, which again has formed the foundation and pre-understanding in which the authors possess. Articles used for this paper was located through the databases/sources shown in table 1.

Table 1. Located Papers Used in This Article

\begin{tabular}{lcccccc}
\hline Database/source & $<2003$ & $\mathbf{2 0 0 4}$ & $\mathbf{2 0 0 5}$ & $\mathbf{2 0 0 6}$ & $\mathbf{2 0 0 7}$ & Total \\
\hline The ACM Portal & 2 & 2 & 0 & 4 & 0 & 8 \\
Emerald & 3 & 0 & 10 & 9 & 2 & 24 \\
ScienceDirect / Elsevier & 8 & 4 & 5 & 7 & 4 & 28 \\
Gartner & 3 & 0 & 17 & 14 & 0 & 34 \\
Google Scholar & 6 & & & & & 6 \\
Waria.com & & & & 8 & & 8 \\
The 2006 BPMS Report & 22 & 6 & 32 & 46 & 6 & 412 \\
Total & 22
\end{tabular}

Due to the authors pre-understanding based on the mentioned books, the search for articles in table 1 is based on a subjective approach where different combinations of words with "Business Process Management" and "agility", "advantage", "ERP", "SOA" etc. to make a more a comprehensive covering of the subject BPM. With in the number of located articles 43 articles were read. Based on the 43 articles 29 keywords were defined which seemed to cover the main characteristics of BPM.

After the preparation of the 29 keywords it was clear that several words were substituted or just synonyms. Therefore the keywords were reduced to 13 concepts in two groups: 1) Agent/rules: Benefits/Motivation/Expectations; BPMS; History; Implementation; Management/Organizational culture; and Maturity, and 2) Processes: Simulation/Modeling; Standardization/Automation; Strategy; Webservices \& SOA; Workflow systems. 
In our search for a framework for BPM we need to find out whether the 43 articles are representative to the total number of articles gathered, which we believe to cover the literature of BPM. In order to document whether this is the case a lexical analysis is used for this purpose. A lexical analysis of all 112 articles was performed using the program Leximancer (http://www.leximancer.com/). Leximancer is a tool to analyse text files, it generates concepts based on context and the frequency of words. Further more the size of each concepts generated indicates how prominent the concepts are in the literature. Using Leximancer now makes it possible to find out what the total number articles is primarily about. But our research is about BPM and therefore we need to look at the generated concepts in relation to BPM, which is also possible through Leximancer. By pre-programming "Business Process Management" as a concept Leximancer makes it possible to see which concepts explains the most of BPM. The result of the lexical analysis is a total number of 84 concepts describing BPM where the four largest concepts in the perspective of BPM explains $63.5 \%$ of the lexical content of BPM. The four major concepts were Management, Technology, Support and Approach. Each of these concepts resulted in a number of quotes where e.g. BPM and Management were described. These quotes were then sorted in relation to keywords and discussed in relation to the overlaying concept.

\section{BUSINESS PROCESS MANAGEMENT}

\subsection{Management in a BPM Perspective}

The most frequent word appearing in the lexical analysis was "management". Management is an often-used word in combination with other management disciplines e.g. Customer Relationship Management, Process management, Supply Chain Management, Content Management, Workflow Management, Knowledge Management etc.

The literature reviewed mostly agree on BPM as a management discipline [8], or a management philosophy [9] and how much companies will benefit from this discipline in gaining more effective leverage of information across their business [10]. The philosophy of BPM compared to earlier management disciplines is that BPM is a journey of continuous process improvement [11] opposite e.g. Business Process Reengineering (BPR) that is only a one time process improvement, and due to that agility and more effective business processes will disappear. BPM is though emerged from earlier management disciplines/theories such as total quality management (TQM), LEAN, Six Sigma, and Business Process Reengineering (BPR) [12]. BPM is about developing management disciplines and deploying technology to be able to handle exceptions in standard procedures so that exceptions becomes standard procedure [13]. To do so the literature also advocates that BPM is used as a formal and structured approach employing governance, methods, policies, metrics, practice and tools to ensure that it defines, manages and continually optimizes its business processes $[14,15]$. The deployment of BPM requires like any other discipline full attention from management, which is often overlooked, the people involved in the 
processes needs day-to-day management [16]. Further more deployment of BPM is different from traditional technology deployments because BPM initiatives is not the IT departments responsibility but the "process management" [17].

Management culture is mentioned as an important factor in having success on BPM, often the emphasis lies on the business processes and the underlying complexity of the technology in how to obtain bottom-line profitability [18, 19]. A wide holistic approach towards BPM as a management discipline and the management commitment to organizational transformation will ensure success [20]. When managing BPM projects the management should also remember the importance of managing the people who work within the processes and how their mutual collaboration can contribute to the quantity of work being done. To be able to manage people in organizations and to set key performance indicators and hereby getting the maximum benefit from BPM, companies need to manage the people interface [21]. Effective management information is necessary for planning and decision making [22].

\subsection{Technology in a BPM Perspective}

Technology in a BPM perspective concerns the crucial importance that technology plays in generating value and underpinning the philosophy of BPM by delivering an infrastructure that drives work though the firm, enabling a regime of monitoring, optimization and traceability [23]. It allows businesses to prepare different scenarios and make the right changes immediately, more effectively and hereby contribute with competitive advantages. Furthermore the technology provides the needed infrastructure to translate strategic choices into concrete plans of action and letting the businesses become more proactive [24].

The objective of BPM is to increase employee and customer value through innovative, flexible and efficient processes but in such a process-managed organizations is more about business transformation then about technology [25]. Simply to view BPM as a label for new technology is a misunderstanding [26] nor an updated version of BPR [27]. Reluctant behavior by thinking of BPM is not surprising while BPM like BPR emphasizes process thinking as a method to lower cost, increase quality of service and improve personal productivity. BPM and BPR are quite similar but the major difference is that BPM is defined as a continuous performance improvement methodology [28] while BPR is not iterative. Both have technology as an enabler but BPM technology at that time in the 1980's did often obstruct process change. In addition the code behind the systems required specialized skills and significant time to change and last, it was proprietary independent of each other applications before the ability to automate the entire process appeared. Today's thinking regarding continual process improvement has significantly changes since the BPR era [29].

The standards as SOA, XML and Web service has all contributed the advance of BPM technology [8] and undertaking a BPM initiative without SOA it is nearly unthinkable [16]. These standards deliver great flexibility and make it a lot easier to invoke complex application processing in BPMS products [8] and often this lack of interoperability can be solved by various web services [18]. BPM and SOA can be 
thought as a new way of linking the business and IT department together however, BPM is fundamentally a top-down approach whereas SOA is a bottom-up [16]. A big technological challenge is therefore to identify the commonalities and resolve the differences [16], and this need can very well be increasing in the future until the BPM technology mature and find its full potential. BPM technology is a new era of software "that uses process models to drive the work through the organization by routing tasks to the relevant employee". This includes a wide range of software spanning from process modeling interfaces, process engines, business rules engines and activity monitoring and analytics components all integrated in a BPM Suite [21].

By the ability to model business processes a process engine is acquired to keep track of the work being done, integrating with third party applications, and hereby ensure later traceability in the processes [22]. Also modeling provides critical capabilities in bringing business intelligence into focused action [9]. The unique about BPM modeling is that changing a process step is as easy as drawing a line between two boxes. This becomes possible because of the models created in a BPMS are linked or integrated directly to the applications and databases by orchestrating the underlying infrastructure completely [11]. Thereby the process engine can be seen as a software server that keeps track of each individual work item and routing it along to the next task when finished [9]. In this context the rule engine becomes a central element in the technology perspective of BPM while it is the place where the business can respond to the continual optimization needs of its processes [23, 24]. In addition it is recommended that these BPM-based applications is frequently updated and finetuned, which means at least once a quarter or even twice as much [15].

BPM has in many cases evolved from a feature of workflow to a flexible way of providing strategic value to the business [18]. Various ways leads to BPM but the "right" choice depend on the leaders understanding of BPM and the commitment to organizational transformation [8]. And the businesses needs to appreciate that BPM technology is a lifelong cycle and not a one-off deployment [25].

\subsection{Support in a BPM Perspective}

The third most frequent word was "Support". The literature emphasizes how Technology/BPM suites support the lifecycle of processes. Supporting lifecycles from vision through modelling, deployment, execution, performance monitoring, and modification is the very core of BPM initiatives [30]. The future BPM systems will support a business transactions/events from the beginning to end and at the same time adding rules and policies needed to support the organizations [27]. Knowledge management, knowledge bases and knowledge sharing is an important factor in obtaining better BPM. The imposing of information flows which support knowledge creation and sharing through out the entire process is a prerequisite for BPM [28]. BPM systems are challenged in how to facilitate and support human-application-level interactions [31] as well as on system-to-system level. Even though many systems support these interactions many BPM system do not accomplish to support human-tohuman requirements as effectively [32] this could be e.g. task handling and sharing information across the organization. Besides the human-human interaction problems, ad hoc processes is yet another kind of processes that sets high demands for BPM 
systems and to the human-human interaction due to the requirements of knowledge and collaboration between workers in such processes. To support Ad hoc processes BPM systems needs to incorporate and support collaboration through shared whiteboards, forums content management capabilities etc. within longer running processes where participants can discuss aspects of the current process [21]. Close collaboration is also needed to support business and IT professionals through the entire process to enhance the amount of control and management of business processes.

Generally the literature mentions BPM suites/systems as a tool to support automation of business decision through a set of business rules that are able to structure loose informal business practices and policies to support processes [30]. The use of business rules can also simplify how processes are developed as well as sharing common processes in different problems [9]. BPM systems also offers automation of repetitive steps in integration application for supporting complex decisions-making [30].

\subsection{Approach in a BPM Perspective}

When talking about approach in a BPM perspective it refers to a broad range of subconcepts with reference to a strategic understanding of the main subject Business Process Management in an EIS perspective. Beyond that the concept "approach" is used in relationship to a broad definition of BPM and issues at the implementation stage. For that reason is seems like there are three main sub-concepts: definition, strategy and implementation, which are linked together by motivating factors in the search for business benefits, agility, flexibility, high performance, optimization, competitive advantages and so on. All words which are typically wrapped around benefits in the context to insure the semantic perception.

Quite often the use and definition of BPM in the literature is visionary and in addition hard to transform into something "easy-to-implement". A more holistic approach is therefore applied as a result of the difficulties in generalization [28]. At the other hand it is claimed that a holistic approach to BPM is needed to embrace all relevant parties and not just to solve pain point in the business or used by the IT department on its own [25]. This difference in the approach gives naturally different perceptions and views of BPM. For instance as a way to standardize business operations in the belief that reducing variation is a viable strategy, or as a method to squeeze cost out inspired by the Business Process Re-engineering theories popular in the 90s [9]. BPM require an approach that fundamental shift from function-centric to process-centric thinking and by that BPM provide visibility into and control over business processes [10]. Despite that the BPM literature is not exhaustive described the scope and definitions it possible to gather [33]. So due to the different perceptions of BPM and the conflicts occurring, understanding BPM might relate in how to use BPM instead of misunderstanding of the potential and the need for process thinking. This lack of process skills in the workforce result in different process management approaches within the entire organization [4] and is for that reason vital to eliminate for receiving the added value to the corporate business thought BPM. Clearness about what the senior management mean of BPM and how they intend to use it is of huge 
importance [28] likewise is the top management commitment and the executive sponsorship [22, 34]. This commitment is essential for receiving success and is crucial to achieve. A further requirement is an alliance between IT and the business leaders to drive a successful process transformation [35].

The approach perspective in the literature selected for this study also embraces a differentiation between different styles in implementing process-enables products like BPM. One strategy is the "pure-play" invented by Gartner Group in 2003 which consists of a holistic application-independent approach there through generic and adaptable technology deals with any business problem [29, 31]. A alternative solution is separately tailor each problem though specific applications supported by many niche applications [31]. The third alternative is a combination between the to worlds by taking the best and by that layering on top of the existent functionality [31]. This combination refers to frameworks, templates, accelerators and are more dependent of vendors terms [31].

Important to keep in mind is that the need for change intervals are getting shorter and shorter to get effective methodologies and get around the business optimization cycle quickly enough [15]. As regards creating a specification of requirements is waste of time $[15,36]$ because nothing stays the same and such a document is not sufficient dynamic. By not acting so can kill a BPM project as it distracts from the critical requirements of proving the efficacy of the BPM approach to the business [36]. Beside that forget all about the waterfall implementation methodology that it is seeking to answer functional requirements [24]. Business leaders often think in reengineering and transforming the organization, but these process-focused technologies is "built-to-change" rather than "built-to-last" [24]. Creating a Center of Excellence (COE) provide a repository for knowledge and best practices development [15] is recommended while the need for coordination and integration increases accordingly BPM projects [22]. Moreover the COE becomes a direct descendant of the steering group and is usually responsible for supporting the BPM projects across the business and keeping momentum going across the organization [22]. Educate users to understand the iterative nature of BPM gives the best chance of success in the initial project [22]. In addition to this the big challenge is to help the business managers to understand that the approach is fundamentally different from the traditionally waterfall or "big bang" implementation approach [31]. The vendors do often describe their support as "round-tripping" with reference to a spiral implementation, which means that their approach is concentrating about analysis, through development, into deployment and then monitoring and optimization [36]. The proposal is to hold short cycles of iterations, which improve the behavior of the application over time and focus on a particular topic, each with "playback" [36]. This methodology ensures flexibility to change quickly as needed and with no surprises emerging [36].

Most organization that has embraced process management has implemented a hybrid or matrix-style approach $[9,28]$. This means that the Line of Business manager (LOB) still run their operations as normal, but for the important process that cross the organizational boundaries is taking care of by a process owner [9]. An effective BPM approach therefore needs a description of the relationships between executives, LOBs, process owners, process architects and process developers [9]. 
Key considerations in the development of a successful BPM projects inchude unambiguous intensions, a business case to gain executive sponsorship, a clear link between BPM - the method and the strategy, acquisition of process competencies, process discipline, improvement and cross-process integration, strategic alignment, culture and leadership, governance, skills and knowledge $[24,28,33]$. Furthermore is the ability to build coalitions and facilitate collaboration among the organization that may have conflicting objectives imperative [4] and the top management commitment [34]. BPM is also about a change in culture and does not success simply through having good systems and the right structure in place [37]. Viewing BPM as "just another development tool" ought to expect conflicts and frustrations [38] and the way to gaining success require a deep understanding of business processes before thinking in implementation a BPM suite [22].

\section{DISCUSSION OF FINDINGS}

A comparison of the keywords from the non-lexical analysis and the keywords (subconcepts) emerged from the lexical analysis are interesting to make to find out whether the read literature are representative to the located literature about BPM. The identified keywords/sub-concepts in the two analyses was compared and in general the two sections of keywords identified are mostly similar to each other. After the execution of the lexical analysis is has now become clearer what the literature actually tells about "benefits" due to that we are able to differentiate what we actually meant by benefits in the non-lexical analysis. The keyword "process" from the non-lexical analysis is typically used in the context and reference to cross-functional possibilities, getting rid of organizational boundaries, understand the information flow or the ability to model the entire infrastructure of the organization. In some cases it is necessary to have insight and knowledge about the foundation of BPM because articles can have the same approach for explaining a topic in BPM. For example talking about knowledge-management, knowledge bases, and knowledge sharing can often be conceived with creating a Centre of Excellence.

On the basis of our research the following sections we will discuss our perception of BPM, where the literature need more research and were have we done the most emerging observations.

Through out this research of the BPM literature we found four major concepts where each of these explaining their perception in relation to BPM. The concept Management cover BPM as a management discipline and methodology where the concept Technology treats more specific relations to architecture and process modelling. These two concepts represent two different parts of what BPM is about where the two other concepts are in between. This makes it difficult to understand what BPM is about. Is it a management discipline or a technology or maybe both? The authors of this article find that BPM can be separated in to two areas: BMP as a management discipline and BPM technology. On this basis we have developed the framework for understanding BPM. 


\subsection{BPM Discipline}

BPM as a management discipline or philosophy is concerning integration of organisational process thinking, as well as keeping management sponsorship etc. BPM is based on management disciplines like Lean, Six Sigma, TQM and BPR, which makes the differences in BPM difficult to clearly define. The literature in our research focuses in the ability to operate agile, make changes immediately and use it as a method to become more proactive to changes. We find that BPM have focus on developing highly flexible business processes as a mean to get competitive advantages. Working with a BPM project in its early stages is in our conviction not different from adopting another management discipline. It is all about changing the organizational culture and to collaborate though the organizational boundaries, getting the management commitment and to settle the project in the entire organization and not just in the IT department. The whole business needs to feel ownership and start working in new ways, which is not special for BPM projects. It is still relevant to educate users and nothing is really new about this. The literature mentions a shift in function-centric thinking to view the entire business though process-centric thinking as an essential starting point for the BPM project. Having the right systems and structure in place does not assure success. Adapting and using BPM to embrace the entire business needs a holistic perception from start. So what is different by using BPM?

The BPM literature often use the word agility to explain one of the benefits by adopting BPM in to the business, but why is the ability to react agile so important and how can it be a competitive advantage is not explicit clarified in the articles but Fingar and Smith are though spending much energy on this topic in the book the Third Wave [1]. We understand agility in relation to BPM as a capability to immediately adapt and react on marked changes. Through interactions with customers, orchestration of internal operations and utilization of the business ecosystems of external business partners [6]. Further we find it of huge importance to be agile and we perceive agility as a prerequisite to BPM. But to use agility in a proactive manner must be done with respect to the business strategy, which can be a challenging balance to manage. The ability of reacting and adapt fast to market changes can be of more importance than be the first to develop a new product. To help the business manage this balance BPM and BPMS are in our opinion inseparable while the technology makes it possible to model the flexible processes and this in coordinance with the business rules.

\subsection{BPM Technology}

BPMS is considered as the practical part and mean to deploy and establish BPM. SOA and web-services are important technologies for creating and establish a BPM environment. Based on these technologies and reference models business process management systems /suites makes it possible to model processes that can directly be executed in to the company. The technology such as BPMS is the foundation for making fully use of the BPM potential. The ability handle hundreds or maybe thousands of processes at the same time or simulate changes in processes and measure 
their impact as well as establishing rules so that processes automatically adapt to changes in the market makes BPMS a truly advantage compared to the systems that we use to day. We perceive that BPMS and the underlying technology in relation to BPM is the mean to make better BPM it can be compared to the 5 principles used in LEAN to make better LEAN.

The vision of having all business people in the organisation modelling their business processes due to their knowledge in their field of expertise seams unlikely but never the less the focus on automating and modelling business processes are of high importance to secure quality, performance and agility. Gartner predicts that the fully potential of BPM won't be accomplished before 2017 [8] due to this we recommend the companies are standing by in investing in new BPMS. EIS systems are already now implementing better process handling which take time not only the development of technology but also the organizations and companies needs to mature to adopt BPM. This doesn't mean that companies shouldn't work with BPM. You don't need technology to make use of BPM but prepare and make the organization mature to think in processes and cross functional.

\subsection{Validity}

The research method used in this paper can be split in to 4 steps or 2 stages: obtaining a pre-understanding, manual reviewing 43 articles, defining keywords on basis of the read articles and preparing the lexical analysis.

To secure consistency in the research each author used the same method in defining keywords. First the keywords were located on basis of what the authors have read (see table 1) hereafter each keyword in every article were ranked according to number of topics and the size of the topics in the articles. This was done independently by each author to secure some sort of objectivity, by not influencing each other. On this basis a comparison of the topics size were made to find out whether the authors had the same perception and strength of keywords in read articles. This comparison was tremendously painless which might be an incident or indicator for the same understanding of the literature, only small adjustments were made.

The lexical analysis serves several purposes, first of all it would be nearly impossible for a human to preserve a complete objective overview of so much literature as covered in this paper and therefore the lexical analysis is a great tool. Further more the lexical analysis is able in a fast way to give an indication of whether the read articles are representative to the rest of the literature and to ensure that the authors haven't chased non-existing topics. The only problem experienced in using a lexical analysis is the usability of the generated output. Programs such as Leximancer are programmed to generate output (quotes) in a context based on mathematical algorithms, but the quality of the contexts are often not as good as hoped, which also have an impact on our conclusion of this research. The profound work in manually analysing the output is very comprehensive but necessary to get quality data about the contents of the literature.

The validity of this research paper must be considered strong due to the wide use of both subjective and objective research methods where a qualitative approach in 
using common human sense together with a quantitative research method through the lexical analysis must be a strong way of ensuring a correct interpretation of the literature. The result of the research is also well documented through the two step analysis first an analysis of the read articles and then an analysis of the entire number of articles.

\section{CONCLUSIONS}

Our main conclusion is that BPM is a practically oriented concept with a weak academic foundation. BPM is a holistic management discipline that uses technology to control and operate the entire business through rules that clearly defines business processes. BPM is about continuous improvement and optimizing processes to ensure high performance and by that achieving agility and flexibility as a tool to gain competitive advantages. We have proposed a framework for BPM which identify a set of conceptual clusters. However we have found no evidence of linkages to the academic field of Management of EIS. The lexical analysis was attempted as a supplementary literature analysis. The combination of a weak conceptual foundation and concept analysis revealed that this approach is less suited to analyze an emerging field. Nevertheless the analysis brought up consistent conceptual clusters.

BPM is obviously a relevant challenge to practice and more research is needed. However future research should make an effort to bridge existing research on EIS and BPM. For space saving reasons tables, references and comparisons have been omitted from this paper. This is available on request in an unabridged paper.

\section{REFERENCES}

1. H. Smith and P. Fingar, Business Process Management: The Third Wave (Meghan-Kiffer Press: Tampa, Fl, 2003).

2. P. Fingar and J. Bellini, The Real-Time Enterprise: Competing on Time with the Revolutionary Business SEx Machine (Meghan-Kiffer Press: USA, 2004).

3. M.A. Dumas, W.M.P. Van der Aalst and A.H.M. Hofstede, Process-aware Information Systems: Bridging People and Software through Process Technology (John Wiley \& Sons, Inc., Hoboken, NJ, 2005).

4. M.J. Melenovsky, Business Process Management as a Discipline. Gartner Research, G00139856 (Gartner Inc., 2006), p.6.

5. T. Schurter, Let's talk about BPM - what it really means (2007). http://www.bpmg.org/Zpost1759.php (Accessed May 16, 2007).

6. V. Sambamurthy, A. Bharadwaj, and V. Grover, Shaping agility through digital options: reconceptualizing the role of information technology in contemporary firms, $M I S$ Quarterly. Volume 27, Number 2, p.237, (2003).

7. C. Møller, ERP II: a conceptual framework for next-generation enterprise systems? Journal of Enterprise Information Management. Volume 18, Number 4, pp.483-497, (2005). 
8. J.B. Hill, J. Sinur, D. Flint, and M.J. Melenovsky, Gartner's Position on Business Process Management, G00136533 (Gartner Inc., 2006), p.26.

9. D. Miers, BPM - Driving Business Performance (BPM Focus Inc., 2006), p.13.

10. M.J. Melenovsky, J. Sinur, J.B. Hill, and D.W. McCoy, Business Process Management: Preparing for the Process-Managed Organization, Gartner Research, G00129461. (Gartner Inc., 2005), p.8.

11. M.J. Melenovsky, Business Process Management Offers a World Where Exceptions No Longer Exist, Gartner Research, G00134777 (Gartner Inc., 2005), p.4.

12. M.J. Melenovsky and K. Harris, Business Process Management Leverages Organizational Knowledge to Create Better Business Value, Gartner Research, G00136713 (Gartner., 2006 ), p.4.

13. D. Miers, BPM-Too Much BP but not Enough of the M. BPM Focus (Lighthouse Point, FL, 2006), p.6.

14. M.J. Melenovsky, Business Process Management's Success Hinges on Business-Led Initiatives, Gartner Research, G00129411 (Gartner Inc., 2005), p.8.

15. D. Miers, Best Practice BPM, ACM Queue. Volume 4, Number 2, pp.40-48, (2006).

16. D. Miers, Issues and Best Practices for the BPM and SOA Journey (BPM Focus Inc., Lighthouse Point, FL, 2006).

17. B. Rosser and R. Buchanan, Business Process Management and Enterprise Architecture: The New Synergy, Gartner Research, G00143201 (Gartner Inc., 2006), p.7.

18. M. Chen, D. Zhang, and L. Zhou, Empowering collaborative commerce with Web services enabled business process management systems, Decision Support Systems. Volume 43, Number 2, pp.530-546, (2007).

19. L. Verner, The Promise and the Challenge, ACM Queue. Volume 4, Number 2, p.9, (2004).

20. M.-A.A. Pantazi and N.B. Georgopoulos, Investigating the impact of business-processcompetent information systems (ISs) on business performance, Managing Service Quality. Volume 16, Number 4, pp.421-434, (2006).

21. D. Miers, Process Innovation and Corporate Agility: Balancing Efficiency and Adaptability in a Knowledge-centric World (BPM Focus Inc., 2006), p.17.

22. D. Miers, The Keys to BPM Project Success (BPM Focus Inc., 2006), p.19.

23. J. Sinur, J.B. Hill and M.J. Melenovsky, Selection Criteria Details for Business Process Management Suites, Gartner Research, G00134657 (Gartner Inc., 2005), p.16.

24. M.J. Melenovsky, J. Sinur, BPM Maturity Model Identifies Six Phases for Successful BPM Adoption, Gartner Research, G00142643 (Gartner Inc., 2006), p.14.

25. P. Redshaw, How Banks Can Benefit from Business Process Management, Gartner Research, G00126515 (2005), p.15.

26. D. Miers, P. Harmon, and C. Hall, The 2006 BPM Suites Report, BPTrends (2006).

27. J. Sinur, Business Process Management Suites Will Be the 'Next Big Thing', Gartner Research, G00125461 (Gartner Inc., 2005), p.6.

28. J.-P. Pritchard and C. Armistead, Business process management - lessons from European business, Business Process Management Journal. Volume 5, Number 1, (1999).

29. J.B. Hill, J. Sinur, D. Flint, and M.J. Melenovsky, Distinguishing Business Process Management From Business Process Re-engineering, Gartner Research, G00136775 (Gartner Inc., 2005), p.5. 
What is Business Process Management: A Two Stage Literature Review of an Emerging

30. D. Miers, P. Harmon, and C. Hall, A Detailed Analysis of BPM Suites, in The 2006 BPM Suites Report Release 2.0, eds. D. Miers, P. Harmon, and C. Hall (2006), p.21.

31. D. Miers, BPM Solution Frameworks: Achieving Revolutionary Objectives through Evolutionary Change. BPM Focus (2006), pp.14-.

32. J. Sinur, D.W. McCoy, and T. Bell, Creating a BPM and Workflow Automation Vendor Checklist, Gartner Research, COM-19-1332 (Gartner Inc., 2003), pp.8.

33. R.G. Lee and B.G. Dale, Business process management: a review and evaluation, Business Process Management Journal. Volume 4, Number3, pp.214-225, (1998).

34. M.A. Mashari, A.A. Mudimigh, and M. Zairi, Enterprise resource planning: A taxonomy of critical factors. European Journal of Operational Research. Volume 146, Number 2, pp.352-364, (2003).

35. J.P. Roberts, How IT Departments Support Business Process Management. Gartner 7 (2006).

36. D. Miers, Getting Past the First BPM Project: Developing a Repeatable BPM Delivery Capability, BPM Focus (2006), pp.17.

37. M. Zairi, Business process management: a boundaryless approach to modern competitiveness, Business Process Management Journal. Volume 3, Number 1, (1997).

38. D.W. McCoy, Re-examine Your Process Mentality to Avoid Business Process Management Pitfalls, Gartner Research, G00127431 (Gartner Inc., 2005), pp.5. 